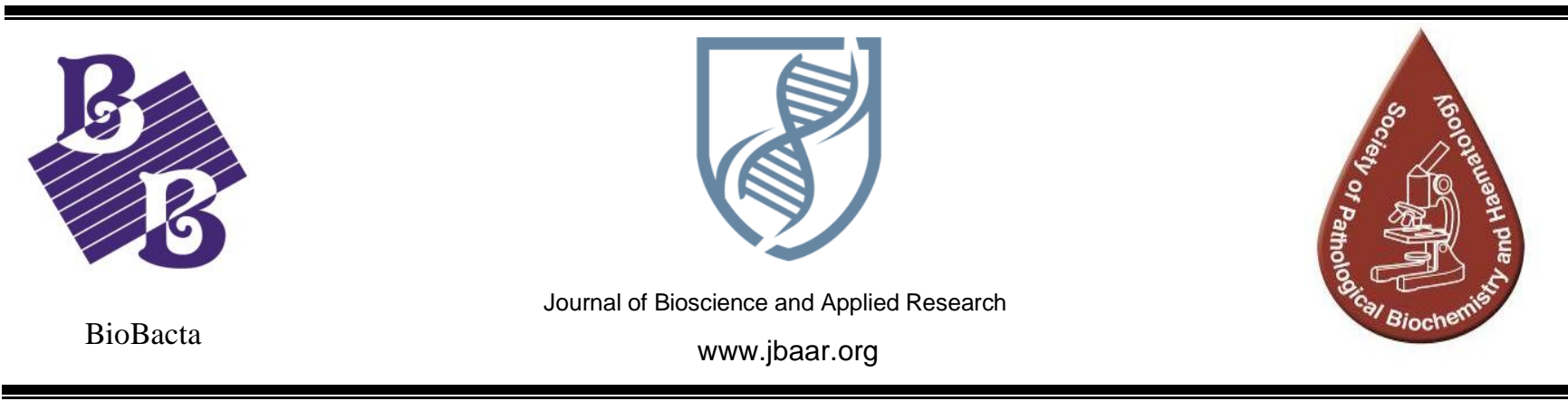

\title{
Molecular expression and single nucleotide polymorphisms of the ILI7A gene among etanercept-treated rheumatoid arthritis patients
}

\author{
Aseel S. Mahmood', Abdul-Kareem A. Al-kazaz', Khadier Z. Mayouf', and Ali H. Ad'hiah, ${ }^{3, *}$ \\ ${ }^{1}$ Biotechnology Department, College of Science, University of Baghdad, Baghdad, Iraq. \\ ${ }^{2}$ College of Medicine, University of Baghdad, Baghdad, Iraq. \\ ${ }^{3}$ Tropical-Biological Research Unit, College of Science, University of Baghdad, Baghdad, Iraq. \\ *Corresponding author (E-mails: dr.a.h.adhiah@gmail.com; dr.ahadhiah@ sc.uobaghdad.edu.iq)
}

DOI: $10.21608 /$ JBAAR.2019.141087

\begin{abstract}
Molecular expression (reverse transcription-quantitative polymerase chain reaction; RT-qPCR) and DNAsequencing-based single nucleotide polymorphisms (SNPs) of interleukin 17A (IL17A) gene were determined in 51 etanercept-treated Iraqi rheumatoid arthritis (RA) patients and 45 control. The results revealed that the relative expression $\left(2^{-\Delta \Delta \mathrm{Ct}}\right)$ of the $I L 17 \mathrm{~A}$ gene was increased by $1.28 \pm 0.29$ fold in RA patients, and such profile was approximated in males $(1.66 \pm 0.58)$ and female $(1.01 \pm 0.28)$ patients. Concerning PCRamplified DNA sequences, out of the 10 encountered SNPs, two SNPs (rs8193038 and rs3819025) showed allele frequencies that exceeded 10\%. The rs8193038 SNP allele and genotype frequencies showed no significant variations between RA patients and control. The second SNP (rs3819025) was observed to have three genotypes (AA, AG, and GG). Among these genotypes, it was observed that the homozygous genotype of the mutant allele (GG) was only recorded in patients with a frequency of $13.7 \%$, while none of the control had this genotype. Such a difference was significant even after the correction of probability $(p c=0.05)$, and the associated OR was 15.34 (95\% C.I.: 1.39 - 169.24). It was also observed that $G$ allele showed a significant increased frequency in patients ( 25.5 vs. $12.2 \%$; OR $=2.46$; $95 \%$ C.I.: $1.14-5.30 ; p=0.015)$, while $A$ allele frequency was significantly decreased $(74.5 v s .87 .8 \%$; OR $=0.41 ; 95 \%$ C.I.: $0.19-0.88 ; p=0.015)$. However, the significance in both cases was lost when the probability was corrected. It was also observed that there was no significant impact of the rs3819025 SNP genotypes on the expression of the IL17A gene. In conclusion, the IL17A gene showed an increased expression in RA patients, and rs3024419 SNP is suggested to be associated with an increased risk to develop the disease in the Iraqi population.
\end{abstract}

Keywords: Rheumatoid arthritis, Interleukin-17A, Gene expression, Single nucleotide polymorphism.

\section{Introduction}

Rheumatoid Arthritis (RA) is a common, systemic, and chronic inflammatory autoimmune disease of the connective tissues. It is characterized by a synovial inflammation of small (hands and feet) and large joints (shoulder and knees). It is a multifactorial and heterogeneous disease, in which both genetic and environmental factors contribute to its etiology, and the interaction(s) between the two factors leads to immunological abnormalities that are involved in its 
pathogenesis (Araki and Mimura, 2016). Among these abnormalities are changed in the profile of cytokines, which are signaling glycoproteins that participate in the regulation of innate and adaptive immune responses. They are suggested to play a significant role in the etiopathogenesis of RA, and cytokines are probably responsible for inflammatory reactions and joint destruction that occur during disease (Mateen et al., 2016).

Interleukin-17A (IL-17A) is one of the cytokines that is regarded to have a role in autoimmune diseases. It is a pro-inflammatory cytokine that is involved in tissue inflammation and destruction through its effects in inducing the expression of pro-inflammatory cytokines (TNF- $\alpha$, IL-1 $\beta$, IL- 6 , and IL-8), which can recruit immune cells (neutrophils, macrophages, and lymphocytes) to the synovium (Kirkham et al., 2014). Animal model studies of arthritis (collagen-induced arthritis) have shown that IL-17-deficient mice and mice treated with anti-IL-17 antibodies demonstrated that IL-17A is crucial in the development of arthritis in these animals by enhancing inflammation of synovium and destruction of joints. It also increases bone destruction by causing an increase in iNOS secretion by RANK and monocyte-CSF-stimulated osteoclasts (Kugyelka et al., 2016).

IL-17A is coded for by a gene (IL17A) located on the short arm of human chromosome 6 (6p12.2), which is a genomic region that harbors HLA genes. Association and family studies have linked this region to RA; therefore, it is possible to consider these genes as potential candidates for the disease (Liu et al., 2016). Studies investigating single nucleotide polymorphisms (SNPs) of the ILI7A gene have confirmed such potential and reported several associations with RA susceptibility, although the observations were not consistent (Lee and Bae, 2017).

The present study was designed to evaluate the gene expression of ILI7A in Iraqi RA patients with some emphasis on clinical, pathological, and laboratory parameters. Also, an intron region of the IL17A gene (chr6:52186276+52186793; 518bp) was amplified and sequenced to inspect SNPs in this region and their association with the disease.

\section{Materials and Methods Patients}

In the beginning, it has to be declared that the present study aimed to enroll RA patients who are newly-diagnosed, but after a period of eight months (November 2015 - June 2016), it was realized that most of the patients, who were referred to the Rheumatology Unit at Baghdad Teaching Hospital, were under therapy; moreover, they were under different therapeutic protocols. Therefore and based on such circumstances and to seek a group of patients that represent a homogenous sample of RA, only patients that received the anti-TNF therapy etanercept for a continuous period of 3-5 years (single weekly subcutaneous dose of $25 \mathrm{mg}$ ) were involved in the study. Accordingly, 51 RA patients (22 males and 29 females) were diagnosed and enrolled in the study and their age range was $20-63$ years. The diagnosis was according to the revised diagnostic criteria established by the American College of Rheumatology (ACR), 2010, which included tender and swollen joint counts, erythrocyte sedimentation rate (ESR), C-reactive protein (CRP), anti-cyclic citrullinated peptide (ACCP) antibodies, rheumatoid factors (RFs) and symptom duration (Aletaha et al., 2010). For comparison, 45 healthy individuals (15 males and 30 females) were also enrolled in the study, and their age range was 25 - 52 years.

The patients were sub-grouped according to some principles; positive and negative for RFs and CRP, and weak $(20.0-39.9 \mathrm{U} / \mathrm{ml})$, moderate $(40.0$ - 59.9 $\mathrm{U} / \mathrm{ml}$ ), and strong ( $\geq 60.0 \mathrm{U} / \mathrm{ml}$ ) positive for ACCP antibodies. A further sub-grouping of patients was based on the Disease Activity Score (DAS)-28. The DAS-28 is a system developed and validated by the EULAR (European League Against Rheumatism) to measure the progress and improvement of RA of patients. The system is based on four assessments, which are TEN28 (number of joints with tenderness upon touching), SW28 (number of swollen joints), ESR, and SA (subjective assessment of disease activity by the patient during the preceding seven days on a scale between 0 and 100). A DAS28 value $>5.1$ corresponds to high disease activity, $3.2-5.1$ corresponds to moderate disease activity and $<3.2$ corresponds to low disease activity (Sengul et al., 2015).

\section{Gene expression of IL17A}

The expression of the $I L 17 A$ gene was determined by the reverse transcription-quantitative polymerase chain reaction (RT-qPCR) method. A ready-to-use reagent (TRIzolTMLS Reagent; Thermo Fischer Scientific; USA) was used to isolate total RNA from blood samples, while the GoTaq ${ }^{\circledR} 1$-Step RT-qPCR System kit (Promega, USA) was used to assess the gene expression and instructions of the manufacturer were followed. Forward and reverse primers for the ILI7A gene (5'-CTCATTGGTGTCACTGCTACTG3' and 5'-CCTGGATTTCGTGGGATTGTG-3', respectively) and the housekeeping gene GAPDH (5'AGCCGAGCCACATCGCT-3' and 5'- 
CAGCCCTGGTGACCAGGC-3', respectively)were adopted according to previously published sequences (Mariaselvam et al., 2014). To determine the expression fold change for the IL17A gene, the $2^{-\Delta \Delta C t}$ was obtained, which represents the Relative Fold Change. Therefore, the results were expressed as a fold change in the expression level of the target gene that was normalized to an endogenous control (housekeeping gene) and relative to the calibrator, which is the target gene in control subjects.

\section{IL17A gene SNPS}

The forward and reverse primers (5' CCAAAATGGTGTCACCCCTGAAC- 3 ' and 5 ', TGCCGTGGGAGAATTATATTAAATCC-3',

respectively) were designed to amplify $518 \mathrm{bp}$ of IL17A intron region (chr6:52186276+52186793) by using the PrimerQuest Tool (https://eu.idtdna.com/PrimerQuest/Home/Index). The genomic DNA was isolated from EDTA blood using the ReliaPrepTM Blood gDNAMiniprep System (Promega Corporation, USA) and subjected to PCR amplification. The PCR reaction was performed in a final volume of $25 \mu \mathrm{l}$, which included $12.5 \mu \mathrm{l}$ GoTaq green Master mix, $0.75 \mu \mathrm{l}$ of each primer $(10 \mu \mathrm{M}), 2$ $\mu 1$ DNA sample $(50 \mathrm{ng}$ ) and $9 \mu \mathrm{l}$ nuclease-free distilled water. The PCR conditions were initial denaturation at $95^{\circ} \mathrm{C}$ for 5 minutes (one cycle), followed by 35 cycles of denaturation at $95^{\circ} \mathrm{C}(30$ seconds), annealing at $60^{\circ} \mathrm{C}$ (30 seconds) and extension at $72^{\circ} \mathrm{C}$ ( 30 seconds), followed by a final extension at $72^{\circ} \mathrm{C}$ for 7 minutes. The amplified PCR fragments were subjected to Sanger's sequencing using an ABI3730XL automated DNA sequencer (Macrogen Corporation - Korea). The genotypes were revealed by the Geneious software version 10.2.2 after alignment with a reference sequence in the Gene Bank.

\section{Statistical analysis}

Data of gene expression were given as mean \pm standard error (SE), and significant differences between means were assessed by ANOVA (Analysis of Variance) followed by either LSD (Least Significant Difference) or Duncan test. In both cases, a probability that equals or less than 0.05 was considered significant. These analyses were carried out through the statistical package SPSS version 13.0.

Allele frequencies of the IL17A gene were estimated by direct gene counting methods, while a significant departure from Hardy-Weinberg equilibrium (HWE) was estimated using the H-W calculator for two alleles.
Genotypes of IL17A SNPs were given as percentage frequencies, and significant differences between their distributions in RA patients and controls were assessed by Fisher's exact probability $(p)$, which was corrected for the number of comparisons that were made at each locus (Bonferroni Correction). Also, the odds ratio (OR) and 95\% confidence interval $(\mathrm{CI})$ were estimated to define the association between a genotype and RA. These estimations were calculated by using the WINPEPI computer programs for epidemiologists.

\section{Results \\ Gene expression}

The relative expression $\left(2^{-\Delta \Delta \mathrm{Ct}}\right)$ of the $I L 17 \mathrm{~A}$ gene was increased by $1.28 \pm 0.29$ fold in RA patients, and such profile was approximated in males $(1.66 \pm 0.58)$ and female $(1.01 \pm 0.28)$ patients. Such expression was subjected to some variation that was related to the subgrouping of patients according to DAS-28 and ACCP antibodies. Low DAS-28 patients showed the lowest mean $(0.11 \pm 0.01)$, while High DAS-28 patients recorded the highest mean $(2.25 \pm 0.66)$, and the difference was significant $(p=0.03)$. For ACCP antibodies, the weak positive patients showed the highest mean $(1.83 \pm 0.53)$, while moderate and strong positive patients showed lower significant means $(0.75 \pm 0.23$ and $0.81 \pm 0.36$, respectively; $p=$ 0.05) (Table 1).

Table 1: Expression fold $\left(2^{-\Delta \Delta C t}\right)$ of ILI7A mRNA in rheumatoid arthritis patients distributed according to laboratory and clinical findings

\begin{tabular}{|c|l|c|c|c|}
\hline \multicolumn{2}{|c|}{ Groups } & $\mathrm{N}$ & $\begin{array}{c}2^{-\Delta \Delta \mathrm{Ct}} \\
(\text { Mean } \pm \text { SE) }\end{array}$ & \multirow{2}{*}{-value } \\
\hline \multirow{2}{*}{$\begin{array}{c}\text { RA } \\
\text { Patients }\end{array}$} & Total & 51 & $1.28 \pm 0.29$ & \\
\cline { 2 - 5 } & Males & 22 & $1.66 \pm 0.58$ & \multirow{2}{*}{ NS } \\
& Females & 29 & $1.01 \pm 0.28$ & \\
\hline $\begin{array}{c}\text { Disease } \\
\text { Duration } \\
\text { (years) }\end{array}$ & $<5$ & 14 & $1.25 \pm 0.56$ & \\
\hline \multirow{3}{*}{ DAS-28 8} & 510 & 26 & $0.81 \pm 0.25$ & NS \\
& Low & 11 & $2.43 \pm 0.94$ & \\
\hline \multirow{2}{*}{ RF } & Medium & 29 & $0.11 \pm 0.01$ & \\
& High & 20 & $2.70 \pm 0.15$ & $\mathbf{0 . 0 3}$ \\
& +ve & 27 & $1.24 \pm 0.66$ & \\
\hline \multirow{2}{*}{ CRP } & -ve & 24 & $1.32 \pm 0.47$ & NS \\
\hline \multirow{2}{*}{ ACCP } & -ve & 33 & $1.06 \pm 0.35$ & \multirow{2}{*}{ NS } \\
& Weak +ve & 24 & $1.69 \pm 0.50$ & \\
& Moderate +ve & 8 & $0.75 \pm 0.53$ & \multirow{2}{*}{$\mathbf{0 . 0 5}$} \\
& Strong +ve & 19 & $0.81 \pm 0.36$ & \\
\hline
\end{tabular}

RA: Rheumatoid arthritis, DAS: Disease activity score, RF: Rheumatoid factors, ACCP: anti-cyclic citrullinated peptide antibodies, +ve: Positive, -ve: Negative, N: Number, $p$ : Probability, NS: Not significant $(p>0.05)$. 


\section{IL17A gene SNPs}

Ten SNPs of IL17A gene were recognized in the amplified region (rs3819025, rs8193037, rs8193038, rs17879568, rs73439726, rs140425841, rs181786431, rs190164861, rs199815459 and rs201292455), but only two SNPs (rs8193038 and rs3819025) were observed to have alleles with polymorphic frequencies. The rs8193038 SNP allele and genotype frequencies showed no significant variations between RA patients and control. The second SNP (rs3819025) was observed to have three genotypes (AA, AG, and GG) in RA patients, while in controls, only AA and AG genotypes were observed. These genotypes were related to two alleles; A and G (Figure 1). Analysis of HWE in RA patients demonstrated a significant departure from it $(p \leq$ 0.01 ), while no significant departure was recorded in control (Table 2).

Among these genotypes and alleles, it was observed that the homozygous genotype of the mutant allele (GG) was only spotted in RA patients with a frequency of $13.7 \%$, while none of the control had this genotype. Such difference was significant even after correction of probability $(p c=0.05)$, and the associated OR was 15.34 (95\% C.I.: 1.39 - 169.24). It was also observed that $G$ allele showed a significant increased frequency in patients $(25.5 \mathrm{vs} .12 .2 \%$; OR = 2.46; 95\% C.I.: 1.14 - 5.30; $p=0.015$ ), while $A$ allele frequency was significantly decreased $(74.5 \mathrm{vs}$. $87.8 \%$; OR $=0.41 ; 95 \%$ C.I.: $0.19-0.88 ; p=0.015$ ). However, the significance in both cases was lost when the probability was corrected (Table 3 ).

\section{SNP impact on IL17A gene expression}

The three genotypes of rs8193038 SNP were inspected for their impact on the expression of the IL17A gene in RA patients. Although patients with GG genotype showed the lowest expression, there was no significant impact of the SNP genotypes on the expression fold of ILI7A mRNA in RA patients (Table 4).

Table 2: observed and expected frequencies of the IL17A gene (rs3819025 SNP) genotypes and their HardyWeinberg equilibrium (HWE) in rheumatoid arthritis patients and controls.

\begin{tabular}{|c|c|c|c|c|}
\hline \multirow{2}{*}{ Genotype } & \multicolumn{2}{|c|}{$\begin{array}{c}\text { RA Patients } \\
(\mathrm{N}=51)\end{array}$} & \multicolumn{2}{c|}{$\begin{array}{c}\text { Control } \\
(\mathrm{N}=45)\end{array}$} \\
\cline { 2 - 5 } & $\mathrm{O}(\%)$ & $\mathrm{E}(\%)$ & $\mathrm{O}(\%)$ & $\mathrm{E}(\%)$ \\
\hline AA & 62.7 & 55.5 & 75.6 & 77.1 \\
\hline AG & 23.5 & 38.0 & 24.4 & 21.6 \\
\hline GG & 13.7 & 6.5 & $\mathrm{ND}$ & 1.3 \\
\hline HWE $p$-value & \multicolumn{2}{|c|}{$<0.01$} & \multicolumn{2}{c|}{$>0.05$} \\
\hline
\end{tabular}

N: Number, O: Observed, E: Expected, $p$ : Probability.
Table 3: Statistical analysis of the association between genotypes and alleles of the IL17A gene (rs3819025 SNP) and rheumatoid arthritis.

\begin{tabular}{|c|c|c|c|c|c|c|c|}
\hline $\begin{array}{c}\text { Genotype } \\
\text { or } \\
\text { Allele }\end{array}$ & \multicolumn{2}{|c|}{$\begin{array}{c}\text { Patients } \\
(\mathrm{N}=51)\end{array}$} & \multicolumn{2}{|c|}{$\begin{array}{c}\text { Controls } \\
(\mathrm{N}=45)\end{array}$} & $\begin{array}{c}\text { Odds } \\
\text { Ratio }\end{array}$ & $\begin{array}{c}95 \% \\
\mathrm{CI}\end{array}$ & $p$ \\
\cline { 2 - 5 } & $\mathrm{N}$ & $\%$ & $\mathrm{~N}$ & $\%$ & & & \\
\hline $\mathrm{AA}$ & 32 & 62.7 & 34 & 75.6 & 0.54 & $\begin{array}{c}0.23- \\
1.31\end{array}$ & $\mathrm{NS}$ \\
\hline $\mathrm{AG}$ & 12 & 23.5 & 11 & 24.4 & 0.95 & $\begin{array}{c}0.38- \\
2.41\end{array}$ & NS \\
\hline $\mathrm{GG}$ & 7 & 13.7 & $\mathrm{ND}$ & $\mathrm{ND}$ & 15.34 & $\begin{array}{c}1.39- \\
169.24\end{array}$ & $\mathbf{0 . 0 0 1}$ \\
\hline$A$ & 76 & 74.5 & 79 & 87.8 & 0.41 & $\begin{array}{c}0.19- \\
0.88\end{array}$ & $\mathbf{0 . 0 1 5}$ \\
\hline$G$ & 26 & 25.5 & 11 & 12.2 & 2.46 & $\begin{array}{c}1.14- \\
5.30\end{array}$ & $\mathbf{0 . 0 1 5}$ \\
\hline
\end{tabular}

$\mathrm{N}$ : Number, CI: Confidence interval, $p$ : Probability, ND: Not detected, NS: Not significant $(p>0.05)$. Significant after correction.

Table 4: Impact of rs3819025 SNP on IL17A mRNA expression in RA patients

\begin{tabular}{|c|c|c|}
\hline Genotype & $\mathrm{N}$ & $2^{-\Delta \Delta \mathrm{Ct}}(\text { Mean } \pm \mathrm{SE})^{*}$ \\
\hline $\mathrm{AA}$ & 32 & $1.46 \pm 0.41^{\mathrm{A}}$ \\
\hline $\mathrm{AG}$ & 12 & $1.28 \pm 0.52^{\mathrm{A}}$ \\
\hline GG & 7 & $0.46 \pm 0.21^{\mathrm{A}}$ \\
\hline
\end{tabular}

$\mathrm{N}$ : Number, *Similar superscript letters represent no significant difference between means $(p>0.05)$.

\section{Discussion}

The assessment of relative IL17A gene expression revealed that total RA patients, as well as male and female patients, showed an increased expression by approximately one-fold. The expression was also influenced by DAS-28, and it showed a gradual increase as patients progressed from Low to High DAS-28. The ACCP antibody status also impacted the expression of the IL17A gene, and weak positive patients showed the highest expression, while moderate and strong positive patients showed a lower expression. These observations suggest that IL-17A might have a role in the pathogenesis of RA, or the expression is subjected to the disease activity (DAS28) and status of ACCP antibodies. Most studies agree with such a theme and up-regulation of IL17A gene expression is positively associated with the development of arthritis (Kugyelka et al., 2016). It was also reported that synovial fluids of RA patients have a high level of IL-17A. These observations have led to the conclusion that through the production of other pro-inflammatory cytokines, IL-17A has a significant, if not a central, role in the pathogenesis of RA (Gaffen, 2009). 


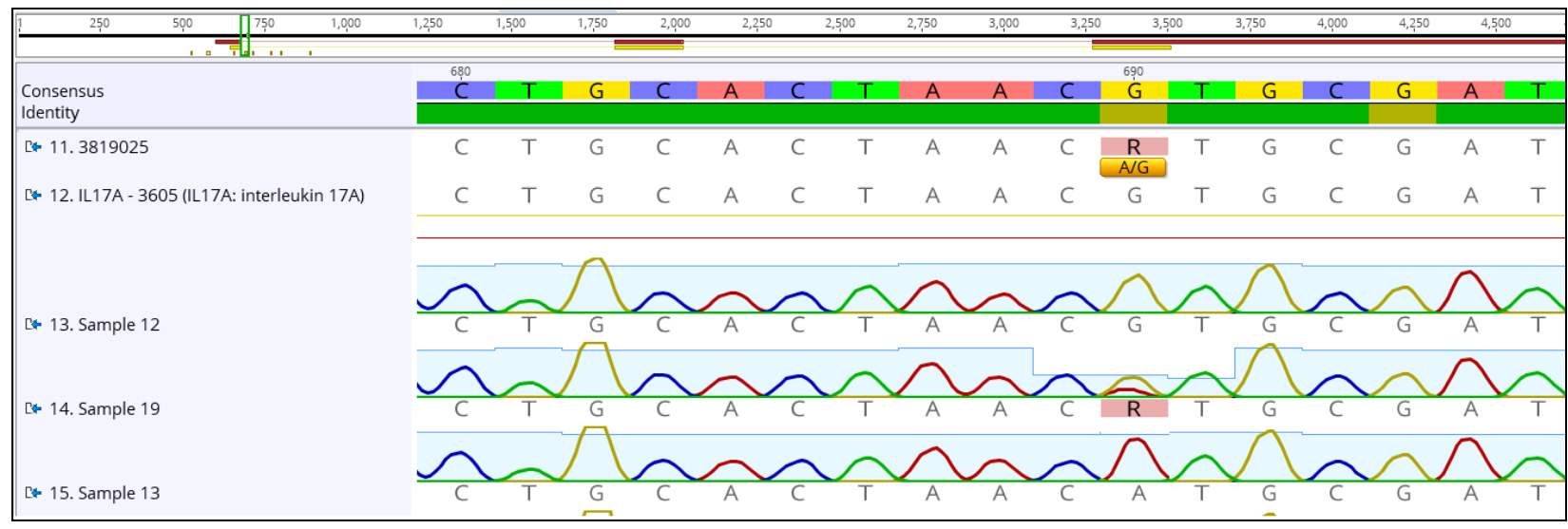

Figure 1: DNA sequence chromatogram of IL17A gene SNP (A/G: rs3819025) showing three genotypes: AA (sample 13), AG (sample 19; R), and GG (sample 12). Also, the reference sequence (rs3819025) is given.

Further investigations revealed that IL-17A is also considered to be important for osteoclastogenic. In an in vitro animal model of osteoclastogenesis, it was found that co-cultured murine osteoblasts and hematopoietic cells treated with IL-17A derived from the synovial fluids of RA patients resulted in increased IL-17A-dependent osteoclastogenesis. Later, it was shown that IL-17A is involved in increased bone resorption in human RA bone explant cultures and enhanced proteoglycan loss from mouse cartilage (Chabaud et al., 2001). Another research group reported that IL-17A promoted osteoclastogenesis in vitro from human CD14+ osteoclast precursors acquired from healthy donors through up-regulation of the receptor activator of NF$\kappa \beta$ (RANK) (Adamopoulos et al., 2010). Such role of IL-17A in the pathogenesis of RA has also been confirmed in animal models of arthritis (CIA). It was found that a blockade of endogenous IL-17A in mice resulted in a suppression of arthritis, and was accompanied by reduced damage of joints, while gene transfer of IL-17A exacerbated CIA (Hashimoto, 2017). Further confirmation has come from a recent Egyptian study, in which RA patients were investigated. Serum level of IL-17A and frequency of Th17 cells were found to be significantly increased in peripheral blood of RA patients. Also, both Th17 cells and serum level of IL-17A were significantly correlated with DAS-28, ESR, CRP, and TNF- $\alpha$ (AlSaadany et al., 2016). Such observations may support the present findings of IL17A role in the etiology and pathogenesis of RA. More recently, the role of IL$17 \mathrm{~A}$ in the pathogenesis of inflammatory arthritis and its implication for clinical practice has been discussed, and it has been concluded that inhibition of IL-17A could be considered as a possible therapeutic strategy for arthritis (Miossec, 2017).
The present study also targeted the role of IL-17A in the etiology of RA through investigating an SNP of such cytokine (rs3819025; A/G). Among the recorded genotypes and alleles, it was observed that the homozygous genotype GG was only observed in patients (13.7\%), while none of the control had this genotype. Such a difference was significant and the associated OR was 15.34. It was also observed that the $G$ allele showed a significantly increased frequency in patients (susceptibility allele), while $A$ allele frequency was significantly decreased (protective allele). Such findings suggest that functional variations of this SNP may contribute to the etiology of RA, and may even promote or protect against RA. Following a similar approach, Shen and colleagues investigated the association between six SNPs of the IL17A gene (rs2275913, rs3819024, rs3819025, rs4711998, rs8193036, and rs8193037) and the risk of RA in a Chinese population (Shen et al., 2015). They found that rs2275913 and rs3819024 SNP variant alleles decrease the risk of RA, while the SNPs rs3819025 and rs8193036 variant alleles increase RA risk of RA. Such findings are in good agreement with the present study results, in which the SNP rs3819025 allele and genotypes showed a significant difference between RA patients and controls. However, the authors of the Chinese study also warranted to determine which of these functional SNPs play pivotal roles in RA and to elucidate the underlying mechanisms of action. No further investigation of this SNP in RA has been carried out, but other SNPs of the IL17A gene have been under intensive studies. In a recent meta-analysis, 14 studies including 3118 RA patients and 2725 controls were enrolled. The analysis revealed a significantly higher serum level of IL-17A in RA patients, and the author presented evidence of associations between the SNPs 
rs2275913, rs763780, and rs3819024 pathogenesis of RA (Lee and Bae, 2017).

In conclusion, the ILI7A gene showed an increased expression in RA patients, and rs3024419 SNP is suggested to be associated with an increased risk to develop the disease in the Iraqi population.

\section{References}

Adamopoulos, I.E., Chao, C., Geissler, R., Laface, D., Blumenschein, W., Iwakura, Y., McClanahan, T., Bowman, E.P., 2010. Interleukin-17A upregulates receptor activator of $\mathrm{NF}-\mathrm{kB}$ on osteoclast precursors. Arthritis Res. Ther. 12, R29. https://doi.org/10.1186/ar2936

Al-Saadany, H.M., Hussein, M.S., Gaber, R.A., Zaytoun, H.A., 2016. Th-17 cells and serum IL17 in rheumatoid arthritis patients: Correlation with disease activity and severity. Egypt. Rheumatol. 38, 1-7. https://doi.org/10.1016/j.ejr.2015.01.001

Aletaha, D., Neogi, T., Silman, A.J., Funovits, J., Felson, D.T., Iii, C.O.B., Birnbaum, N.S., Burmester, G.R., Bykerk, V.P., Cohen, M.D., Combe, B., Costenbader, K.H., Dougados, M., Emery, P., Ferraccioli, G., Hazes, J.M.W., Hobbs, K., Huizinga, T.W.J., Kavanaugh, A., Kay, J., Kvien, T.K., Laing, T., Mease, P., Ménard, H. a, Moreland, L.W., Naden, R.L., Pincus, T., Smolen, J.S., Stanislawska-biernat, E., Symmons, D., Tak, P.P., Upchurch, K.S., Vencovský, J., Wolfe, F., Hawker, G., 2010. 2010 Rheumatoid arthritis classifi cation criteria : an American College of Rheumatology / European League Against Rheumatism collaborative initiative. Ann. Rheum. Dis. 69, 1580-1588. https://doi.org/10.1136/ard.2010.138461

Araki, Y., Mimura, T., 2016. The mechanisms underlying chronic inflammation in rheumatoid arthritis from the perspective of the epigenetic landscape. J. Immunol. Res. 2016, 10 pages. https://doi.org/10.1155/2016/6290682

Chabaud, M., Lubberts, E., Joosten, L., Berg, W. Van Den, Miossec, P., 2001. IL-17 derived from juxta-articular bone and synovium contributes to joint degradation in rheumatoid arthritis. Arthritis Res. 3, 168-177.

Gaffen, S.L., 2009. The role of interleukin-17 in the pathogenesis of rheumatoid arthritis. Curr. Rheumatol. Rep. 11, 365-70.

Hashimoto, M., 2017. Th17 in Animal Models of Rheumatoid Arthritis. J. Clin. Med. 6, 73. https://doi.org/10.3390/jcm6070073
,Wavanaugh, A., Reich, K., 2014. Interleukin-17A: a unique pathway in immunemediated diseases: psoriasis, psoriatic arthritis and rheumatoid arthritis. Immunology 141, 13342. https://doi.org/10.1111/imm.12142

Kugyelka, R., Kohl, Z., Olasz, K., Mikecz, K., Rauch, T.A., Glant, T.T., Boldizsar, F., 2016. Enigma of IL-17 and Th17 cells in rheumatoid arthritis and in autoimmune animal models of arthritis. Mediators Inflamm. 2016, 1-11. https://doi.org/10.1155/2016/6145810

Lee, Y.H., Bae, S.-C., 2017. Associations between circulating IL-17 levels and rheumatoid arthritis and between IL-17 gene polymorphisms and disease susceptibility: a meta-analysis. Postgrad. Med. J. 93, 465-471. https://doi.org/10.1136/postgradmedj-2016134637

Liu, W.-X., Jiang, Y., Hu, Q.-X., You, X.-B., 2016. HLA-DRB1 shared epitope allele polymorphisms and rheumatoid arthritis: a systemic review and meta-analysis. Clin. Invest. Med. 39, E182-E203.

Mariaselvam, C.M., Aoki, M., Salah, S., Boukouaci, W., Moins-Teisserenc, H., Charron, D., Krishnamoorthy, R., Tamouza, R., Negi, V.S., 2014. Cytokine expression and cytokine-based T cell profiling in South Indian rheumatoid arthritis. Immunobiology 219, 772-777. https://doi.org/10.1016/j.imbio.2014.06.004

Mateen, S., Zafar, A., Moin, S., Khan, A.Q., Zubair, S., 2016. Understanding the role of cytokines in the pathogenesis of rheumatoid arthritis. Clin. Chim. Acta 455, 161-171. https://doi.org/10.1016/j.cca.2016.02.010

Miossec, P., 2017. Update on interleukin-17: a role in the pathogenesis of inflammatory arthritis and implication for clinical practice. RMD Open 3, e000284. https://doi.org/10.1136/rmdopen-2016000284

Sengul, I., Akcay-Yalbuzdag, S., Ince, B., GokselKaratepe, A., Kaya, T., 2015. Comparison of the DAS28-CRP and DAS28-ESR in patients with rheumatoid arthritis. Int. J. Rheum. Dis. 18, 640-645. https://doi.org/10.1111/1756$185 X .12695$

Shen, L., Zhang, H., Yan, T., Zhou, G., Liu, R., 2015. Association between interleukin 17A polymorphisms and susceptibility to rheumatoid arthritis in a Chinese population. Gene 566, 1822. https://doi.org/10.1016/j.gene.2015.04.028 